\title{
WIR HABEN UNS FÜR EINEN ANDEREN KANDIDATEN ENTSCHIEDEN - ZU DEN ARGUMENTATIONSMUSTERN IN DEN DEUTSCHEN ABSAGESCHREIBEN AUF BEWERBUNGEN
}

\section{ABSTRACT: Argumentation patterns in German job application refusal letters}

The subject of this analysis are German job application refusal letters, in which a number of structural elements can be distinguished. One of them is argumentation or justification. This text studies logical argumentation strategies and its objective is to create a typology of justifications in job application refusal letters.

KEYWORDS: refusal letters, argumentation, topos, logical argumentation

„Argumentieren ist eine der Formen, Konflikte, Meinungsverschiedenheiten in unserem Alltag zu lösen und auszugleichen“" (Sáfár, 1999, S. 103 f.). Diese gehören jedoch zu alltäglichen Erscheinungen, auf die man nicht immer richtig zu reagieren weiß. Der Einsatz der logischen Argumentation wird dabei als ,goldene Mitte“ empfohlen, denn im Falle der „konfliktiven Sprechakte“ (vgl. Unwerth \& Buschmann, 1981) ist die Leistung der Argumentation unverkennbar. Sie wird nämlich als ,Übergang vom Emotionalen zu Kognitiv-Rationalem, zur logischen Klarheit“" (Sáfár, 1999, S. 104) betrachtet.

Die logische Argumentation wird im Falle der ablehnenden Antworten auf Bewerbungen besonders erwartet, zumal diese zu den konflikterregenden Reaktionen gehören. Die Bewerber dagegen können aus den angeführten Begründungen Schlüsse in Bezug auf weitere Bewerbung ziehen, ihre eventuellen Bewerbungsfehler einsehen und schließlich die Qualität des eigenen Sich-Bewerbens steigern, wodurch ihre Bemühungen in Zukunft mit Erfolg gekrönt werden könnten.

Gerade die Analyse von Strategien der angeblich logischen ${ }^{1}$ Argumentation in deutschen authentischen Absageschreiben auf Bewerbungen ist Gegenstand des vorliegen-

Joanna Szczęk - Uniwersytet Wrocławski, Wrocław, joanna.szczek@uwr.edu.pl

${ }^{1}$ So wird es seitens der potentiellen Arbeitgeber betrachtet. 
den Beitrags. Die Untersuchung erfolgt in Anlehnung an die argumentationsanalytische Methode von Wengeler (2003)2. Mein Hauptanliegen ist zu zeigen, welche Topoi sich in den Begründungen in den ablehnenden Antworten auf Bewerbungen unterscheiden lassen, was wiederum ermöglicht, die behandelte Textsorte, sowohl in thematischer als auch in struktureller Hinsicht, besser zu bestimmen.

\section{Untersuchungskorpus}

Das Analysekorpus für die vorliegende Studie stellen 253 authentische deutsche Absageschreiben auf Bewerbungen dar. Sie wurden in den Jahren 2001-2012 gesammelt.

Absageschreiben auf Bewerbungen werden als Texte definiert, die 1. auf der Grundlage einer schriftlichen Bewerbung ${ }^{3}$ 2. von einem Kommunikationspartner (= Bewerber) an 3. einen anderen Kommunikationspartner ( $=$ den potentiellen Arbeitgeber) gerichtet sind; die 4. von diesem anderen Kommunikationspartner (= dem potentiellen Arbeitgeber) verfasst werden und 5. eine negative Entscheidung in Bezug auf die eingereichte Bewerbung enthalten (Szczęk, 2015, S. 48).

In Bezug auf ihre Struktur sowie die in diesen mitgeteilte Intention des Senders (= des Absageschreibers) und den damit verbundenen Typ der ablehnenden Antwort werden im Korpus drei Typen von Absageschreiben unterschieden ${ }^{4}$ :

- Aperitif-Briefe, die nur die kurze Bestätigung des Eingangs der Bewerbungsunterlagen und die Information über deren weitere Bearbeitung enthalten ${ }^{5}$; im Korpus finden sich dazu 16 Beispiele;

- eigentliche Absageschreiben, in denen die Ablehnung/Absage in Bezug auf die eingereichte Bewerbung ausgedrückt wird; im Korpus finden sich dazu 232 Beispiele;

- Eisschreiben, in denen dem Bewerber zwar abgesagt, aber zugleich mitgeteilt wird, dass seine Unterlagen für „spätere Wiederverwendung“ ${ }^{\text {“6 }}$ vorgemerkt und gespeichert werden ${ }^{7}$; im Korpus finden sich dazu 5 Beispiele.

Die gesammelten Beispiele stammen von zwei Bewerberinnen und zwei Bewerbern, die in verschiedenen Teilen Deutschlands wohnen. Es handelt sich überwiegend um

${ }^{2}$ Vgl. hierzu auch Dąbrowska-Burkhardt (2012).

${ }^{3}$ Hier verstanden als Angebot.

${ }^{4}$ Vgl. hierzu auch Szczęk (2015, S. 32).

${ }^{5}$ Dadurch wird der Appetit auf die evtl. künftige Einstellung angeregt, wodurch Aperitif-Briefe in ihrer Funktion dem eigentlichen Aperitif ähneln, der als „,appetitanregendes alkoholisches Getränk“ definiert wird, vgl. Duden online: http://www.duden.de/rechtschreibung/Aperitif [Zugriff: 14.11.2015].

${ }^{6}$ Authentisches Zitat aus einem Eisschreiben.

${ }^{7}$ Diese Bezeichnung ähnelt der deutschen Redewendung jdn. aufs Eis legen, die bedeutet: salopp: ,jemanden [vorläufig] zurückstellen; jemanden für spätere Verwendung vormerken“, vgl. Duden online: http://www.duden.de/rechtschreibung/Eis_Wasser_Speise [Zugriff: 14.11.2015]. 
Dauerarbeitslose, die auf der Suche nach einer Stelle waren. Das Alter der Bewerber beträgt zwischen 36-50 Jahren.

In Bezug auf die Absender der Absageschreiben handelt es sich um fast alle möglichen und denkbaren Institutionen, an die sich die Bewerber gewandt haben, in denen einem Arbeitslosen eine geistige Tätigkeit angeboten werden könnte.

\section{Absageschreiben - einige Bemerkungen zur Textsorte}

Absageschreiben als solche sind eine in der Textlinguistik ${ }^{8}$ bekannte, jedoch eher seltener behandelte Textsorte. Sie tauchen in den einschlägigen Textsortenklassifikationen und -typologien kaum auf.

In Bezug auf deren kommunikativen Wert sind sie als Texte zu betrachten, in denen ablehnende Antworten auf gewisse Stimuli initiativen Charakters erteilt werden. Als Stimuli gelten hier die Bewerbungsunterlagen - Angebote seitens des Bewerbers. Demgemäß sind Absageschreiben reaktiven oder respondierenden ${ }^{9}$ Charakters, da deren Autoren einen Dissens mit dem in dem Bewerbungsschreiben enthaltenen Angebot ausdrücken.

Als die Hauptillokution in den Absageschreiben kann man ABLEHNEN/ABSAGEN bestimmen, was auch Drescher (1994, S. 129) bestätigt, indem sie feststellt, dass ,die Absage als zentrale inhaltliche Komponente die kommunikative Funktion des Briefes realisiert".

Neben der Hauptillokution können in den behandelten Beispielen auch andere Illokutionen identifiziert werden, wodurch die Struktur der Absageschreiben als komplex beschrieben werden kann. Dies reiht sich in das Konzept des Textes von Brinker (2010, S. 85) ein, der den Text ,als hierarchisch strukturierte Abfolge von elementaren sprachlichen Handlungen" auffasst, und das Modell von Schröder (2003), dessen Kern die These bildet, dass ,sich die Texthandlung als komplexe Handlung beschreiben lässt, da sie eine Handlung ist und aus mehreren aufeinander folgenden und zusammenhängenden Teilhandlungen besteht". Dabei wird hervorgehoben, dass es sich um eine Teil-Ganzes-Beziehung handelt, im Rahmen deren Teilhandlungen ,einen Beitrag zum Vollzug der Gesamthandlung leisten“ (Czachur, 2007, S. 70). ABLEHNEN/ABSAGEN bildet daher den Kern jedes Absageschreibens, und „verweist auf das Globalziel des Textes [...]" (ebd.), wodurch auch die Funktion dieser Texte bestimmt wird.

Dieses schlägt sich auch in der Struktur der Absageschreiben nieder, die es möglich macht, konventionalisierte Muster ${ }^{10}$ der besprochenen Textsorte zu erstellen:

${ }^{8}$ Vgl. z. B. Drescher (1994), Gałczyńska (2003), Itakura (2001, 2004), Müller \& Moser (2006), Wierzbicka (1987), Hayashi (1997), Duden (2005a, 2005b) u. a.

${ }^{9}$ Diese Bezeichnung bezieht sich eher auf den mündlichen Gebrauch.

${ }^{10}$ Vgl. Lüger (1992). 
Tab. 1: Textmuster für Absageschreiben (Szczęk, 2015, S. 189)

\section{Aperitif-Briefe}

1. Eröffnung: faktografische Informationen

2. Hauptteil:

a. Bestätigungsformel:

- Ausdruck der Freude, dass man sich bei der jeweiligen Firma beworben hat

- Bestätigung des Eingangs der Bewerbungsunterlagen

- Dank für die Zusendung der Unterlagen

b. Information über das Auswahlverfahren:

- lange Dauer der Prüfung der Unterlagen

- noch nicht begonnenes Auswahlverfahren

- sukzessive Auswertung der Unterlagen

- Weiterleitung der Unterlagen

c. Bitte um Geduld

d. Aperitif - Versprechung einer späteren Kontaktaufnahme

e. Information über die Speicherung der Daten

3. Schluss: Grußzeile und Unterschrift
Eigentliche Absageschreiben

und Eisschreiben

1. Eröffnung: faktografische Informationen

2. Hauptteil:

a. Anrede

b. Bestätigungsformel:

- Feststellung der Tatsache, dass man sich beworben hat

- Bestätigung und Dank für die Bewerbung

- Dank für das Interesse an der Arbeit

c. Beschreibung der Auswahlrituale, des Auswahlverfahrens

d. Formulieren der Absage + evtl. Vormerkung $(=, \text {,Aufs-Eis-Legen") })^{11}$

e. Nennen der Absagegründe

f. Entschuldigung des potenziellen Arbeitgebers für die Erteilung der Absage

g. Trost

h. Bitte um Verständnis

i. Dank für die Mühe, die man sich bei der Anfertigung der Bewerbung gegeben hat

j. Gutgemeinte Ratschläge für die weitere Arbeitssuche

k. Ausdruck des Bedauerns

1. Wünsche für die weitere Arbeitssuche

$\mathrm{m}$. Rücksendung der eingereichten Unterlagen

3. Schluss: Grußzeile und Unterschrift

4. Anlagenvermerk (nicht obligatorisch)

\section{Zur Argumentation in Absageschreiben auf Bewerbungen}

Das Absagen gehört zu den konfliktiven Sprechakten, die unterschiedliche Folgen nach sich ziehen und v. a. das Gleichgewicht zwischen den Interaktanten gefährden können. Daher ist die Rolle der Argumentation in diesem Kontext besonders hervorzuheben. Wenn man dieses auf die Kommunikationssituation in den Absageschreiben überträgt, erkennt man nämlich, dass die Autoren der Absageschreiben bemüht sind, die Bewerber - trotz Absage - aufzuwerten, in ihrem großen Wert und ihrer Nützlichkeit für den Arbeitsmarkt zu bestätigen, und dadurch vielleicht auch den künftigen „Konflikten“ vorzubeugen, wie man es auch in den Ratschlägen fürs Absagen unterstreicht:

\footnotetext{
${ }^{11}$ Dies nur in den Eisschreiben.
} 
Wenn Unternehmen Bewerbern absagen müssen, dann tun sie das meist gestelzt, etwas plump und barsch. Aber sie können auch anders - und sorgen mit freundlichen oder sogar originellen Briefen für einen Tiefkühl-Vorrat guter Kandidaten. Den Firmen geht es nicht allein darum, den ohnehin enttäuschten Bewerbern Formulierungen im Kasernenhofton oder Beamtendeutsch zu ersparen. Sie wollen auch vermeiden, gute Kandidaten ohne Not zu verprellen. Denn im Leben trifft man sich immer zweimal ${ }^{12}$.

Von Herbig (1992, S. 62) wird ARGUMENTIEREN als ein Handlungstyp betrachtet und folgendermaßen definiert:

[...] ein komplexer Handlungstyp, mit dessen Hilfe ein Sprecher aufgrund seiner Situationseinschätzung den für einen oder mehrere Adressaten erkennbaren Versuch unternimmt, diese(n) mit Hilfe einer oder mehrerer unterstützender Äußerungen zu bewegen, tatsächliche oder nur der Situationseinschätzung nach „strittige“ Geltungsansprüche der Wahrheit oder Richtigkeit von Äußerungen zu akzeptieren ${ }^{13}$. (Herbig, 1992, S. 62, Hervorhebung - J.S.)

Sprechhandlungen, die das Argumentieren begleiten und somit zu dessen Komplexität beitragen, sind dem Handlungstyp ERKLÄREN-WARUM zuzuordnen, Sprechhandlungen, bei denen das Zustandekommen eines Sachverhalts expliziert wird, wiederum den Handlungstypen RECHTFERTIGEN und BEGRÜNDEN.

\subsection{Zum Sprechakt BEGRÜNDEN in Absageschreiben}

In den Absageschreiben wird die erteilte Absage mit dem Sprechakt BEGRÜNDEN untermauert. Begründungen ,gehören [nämlich - J. S.] zum festen Bestandteil alltäglichen gesellschaftlichen Handelns" (Gohl, 2006, S. 181). Ehlich \& Rehbein (1986, S. 89) definieren Begründen „als eine Form sprachlichen Handelns [...], das als ,Element des Alltagswissens und der alltäglichen gesellschaftlichen Praxis` in ganz verschiedenen kommunikativen Zusammenhängen eine Rolle spielt" und präziseren es folgendermaßen:

Das Begründen stellt also die Handlung eines Sprechers dar, deren Resultat eine Veränderung im Wissen des Hörers in Bezug auf die Handlungserfordernis ist. Der ,Punkt ${ }^{\star}$ der Illokution ist bei dieser Handlung also nicht schon darin realisiert, dass ein Sprecher eine sprachliche Handlung als ,Begründung' deklariert oder als solche beabsichtigt, sondern er ist erst erreicht, wenn und sofern diese Veränderung der Wissenszustände beim Hörer erreicht ist. (Ehlich \& Rehbein, 1986, S. 94)

Dieser Handlung werden demgemäß folgende Merkmale zugeschrieben (Gohl, 2006, S. 49):

${ }^{12}$ Vgl. http://www.manager-magazin.de/koepfe/karriere/0,2828,321132,00.html [Zugriff: 4.11.2007].

${ }^{13}$ Vgl. hierzu die Definition der Argumentation von Perelman (1979, S. 328): ,nichtformale Beweisführung, die darauf abzielt, die Zustimmung durch eine Zuhörerschaft hervorzurufen oder zu bekräftigen“. 
- Sinnbezogenheit $\rightarrow$ Veränderung des Wissens beim Hörer,

- Orientierung auf ein Gegenüber $\rightarrow$ Gerichtetheit des Begründens auf den Interaktanten,

- Objektivität des Sinnbezugs $\rightarrow$ Kenntlichmachen der Begründung für die anderen Interagierenden,

- Routinisierung und Sedimentierung $\rightarrow$ Herausbildung sprachlicher Praktiken,

- die Rolle der Anderen bei der Konstitution der Handlung.

Die am häufigsten gebrauchten Mittel, mit denen eine Begründung zum Ausdruck gebracht wird, sind v. a. kausale Konstruktionen mit den Konjunktionen weil, da, denn, dass oder mit der Präposition wegen.

In den für die Zwecke des vorliegenden Beitrags untersuchten Absageschreiben findet man den strukturell-thematischen Teil, in dem die Begründung für die erteilte Absage angeführt wird. Dieser kommt dabei die Aufgabe zu, die getroffene - hier negative - Entscheidung mit Argumenten zu belegen, um einem möglichen Konflikt vorzubeugen und eventuelle negative Folgen zu minimieren, denn

[...] cechą charakterystyczną wypowiedzi zawierających odmowę jest występowanie zabiegów interpersonalnych mających złagodzić jej znaczenie. Trzeba pamiętać, że odmowa jest „ostrym” posunięciem interpersonalnym, niesie zapowiedź blokady działania, o które zabiegał partner i na którym mu zależało. (Nęcki, 1996, S. 115 f.)

Die Begründung wird entweder nach der ABLEHNUNG/ABSAGE oder aus strategischen Gründen - ganz am Anfang im brieföffnenden Teil, direkt nach der Dankesformel und vor dem ABLEHNEN/ABSAGEN platziert. Dies hat nämlich den Vorteil, dass der Bewerber schon in den ersten Zeilen des Antwortschreibens auf die Erteilung der Absage vorbereitet wird. Diese Strategie kann demgemäß als eine Art „Vorsichtsmaßnahme" aufgefasst werden (vgl. Szczęk, 2015, S. 276).

Das weitere Ziel ist es, die Aussagekraft der Ablehnung zu mildern und ein positives Verhältnis zu dem Empfänger aufzubauen (vgl. Szczęk, 2015).

Interessant ist es zu erwähnen, dass man in der Ratgeber-Literatur in Bezug auf das Vorkommen der Begründung in Absageschreiben unterschiedliche Meinungen findet. Einerseits wird nämlich hervorgehoben, dass eine Begründung ein notwendiger Teil der Absage ist, wie z. B. im Duden (1997; 2005a, S. 239 ff.; 2005b, S. 129 ff.), wobei drei Möglichkeiten genannt werden, mit Absagen geschickt umzugehen: „Dem Bewerber mitteilen, dass er zwar in die engere Wahl gekommen ist, aber die Wahl schließlich auf einen anderen fiel“; oder ,dem Bewerber mitteilen, dass er den Anforderungen der ausgeschriebenen Stelle voll entsprach, aber seine Gehaltsvorstellungen über den Etat für diese Stelle hinausgehen“; oder „dem Bewerber erklären, dass es mehrere Interessenten mit hoher Qualifikation gab, einer von ihnen jedoch bereits über einschlägige Erfahrungen im ausgeschriebenen Arbeitsbereich verfügt". Die oben nach Duden erwähnten Formulierungen können dann als Strategien des Absagens betrachtet werden. 
Andererseits wird ganz offiziell davor gewarnt, wie z. B. bei Hovermann (2009, S. 79): „Vermeiden Sie daher möglichst eine Rechtfertigung Ihrer Entscheidung. Die Begründung für eine Ablehnung könnte als Diskriminierung angesehen werden“.

\section{Argumentationsmuster in Absageschreiben - Analyse des Materials}

Argumentationsmuster werden als ,wiederkehrende Aussagen in einem großen Textkorpus zu einem öffentlichen Themenbereich verstanden [...]“ (Dąbrowska-Burkhardt, 2012, S. 35).

In den Absageschreiben lassen sich folgende Muster unterscheiden:

1. Muster 1: Wir stellen einfach nicht ein, z. B.: wir nehmen keine Einstellung vor; Leider ist es nicht möglich, Ihrer Bewerbung zu entsprechen; weil wir zur Zeit keine Einstellung vornehmen;

2. Muster 2: Es gibt keine freie Stelle, z. B.: wir haben auch in absehbarer Zeit keine Stelle frei; Wir sagen Ihnen in Ihrem Fall nicht gerne ab, können Ihnen jedoch keine Position in unserem Unternehmen anbieten; Wir haben auch in absehbarer Zeit keine Stelle frei; Wir nehmen keine Einstellung vor; aufgrund fehlender Vakanzen;

- Untermuster 2a: Wir haben keine Stelle, die den Qualifikationen des Bewerbers entspricht, z. B.: es steht uns keine Stelle zur Verfügung, die wir Ihnen im Hinblick auf Ihre Qualifikationen anbieten könnten; mangels entsprechender Stellen; Zur Zeit ist keine Ihren Qualifikationen entsprechende Position vorhanden; Leider muss ich Ihnen mitteilen, dass mangels entsprechender Stellen keine Möglichkeit besteht, Sie hier zu beschäftigen; wir haben keine Ihrer Qualifikation entsprechende freie Stelle zu besetzen, so dass Ihrer Bewerbung nicht näher getreten werden kann; derzeit sehen wir jedoch keine Einsatzmöglichkeiten, die Ihrem Profil entsprechen würden; Leider kann ich Ihnen heute nur mitteilen, dass wir zur Zeit und auch in absehbar keine adäquate Stelle im Haus frei haben; In meiner Stelle ergibt sich jedoch in absehbarer Zeit keine für Sie geeignete Beschäftigungsmöglichkeit; Nach eingehender Prüfung Ihrer Bewerbung bin ich zu dem Ergebnis gekommen, dass ich Ihnen kein Angebot in unserem Haus machen kann; Zu unserem Bedauern müssen wir Ihnen mitteilen, dass wir Ihnen keine Ihren Fähigkeiten und Wünschen entsprechende Stelle anbieten können; Auch unter Würdigung des von Ihnen dargelegten qualifizierten beruflichen Werdegangs [...] muss ich Ihnen mitteilen, dass ich Ihnen zur Zeit und in absehbarer Zeit kein Ihren Wünschen und Ihrer Qualifikation entsprechendes Stellenangebot unterbreiten kann;

- Untermuster 2b: Die Stellen, die dem Profil des Bewerbers evtl. entsprechen würden, sind besetzt, z. B.: da die Ihrer beruflichen Entwicklung und Qualifikation entsprechenden Arbeitsplätze besetzt sind, sehen wir leider keine Möglichkeit, Ihnen ein für Sie interessantes Angebot zu unterbreiten. 
- Untermuster 2c: Es gibt Personalüberhang, z. B.: auf Grund des bestehenden Personalüberhanges derzeit für Bewerber/innen, die nicht dauerhaft bei [...] beschäftigt sind, keine Einstellungsmöglichkeit besteht.

- Untermuster 2d: Es können keine neuen Plätze geschaffen werden, z.B.: Leider sehen wir im Moment auch keine Möglichkeit, zusätzliche Stellen in unserem Haus zu schaffen;

3. Muster 3: Wir stellen aus wirtschaftlichen Gründen nicht ein, z. B.: Aus personalwirtschaftlichen Gründen können wir leider keine Einstellungen vornehmen; aufgrund struktureller und personalwirtschaftlicher Maßnahmen; aufgrund der dramatischen Haushaltslage; Auf Grund der dramatischen Haushaltslage des Landes [...] ist es in diesem Jahr zu drastischen Einsparungen im Bereich der Personalmittel gekommen. Dieser massive Stellenabbau wird sich auch in den kommenden Haushaltsjahren fortsetzen. Im Haushaltsentlastungsgesetz wurde nun festgelegt, dass Nachbesetzungen von Stellen im Wege von Neueinstellungen grundsätzlich nicht möglich sind. Ich sehe daher keine Möglichkeit Ihre Bewerbung bei Stellenbesetzungen zu berücksichtigen.

- Untermuster 3a: Es gibt Einstellungsstopp, z. B.: wegen bestehenden Einstellungsstopps; da es uns wegen des bestehenden Einstellungsstopps nicht möglich ist, Ihnen den gewünschten Arbeitsplatz zur Verfügung zu stellen;

- Untermuster 3b: Die Stellen werden abgebaut, der Betrieb wird umstrukturiert, z. B.: aufgrund des fortgesetzten Stellenabbaus; aufgrund betrieblicher Umstrukturierung; nach weiteren Etatkürzungen sehen wir uns gezwungen, Einsparungen vorzunehmen; wir müssen Ihnen nunmehr leider mitteilen, dass wir trotz Prüfung Ihrer Unterlagen und auf Grund betrieblicher Umstrukturierung keine externen Bewerbungen berücksichtigen können; auf Grund struktureller und personalwirtschaftlicher Maßnahmen, zum gegenwärtigen Zeitpunkt generell keine Neueinstellungen vorgenommen werden; im Personalbereich sind umfangreiche Stellenreduzierungen umzusetzen, wodurch ein erheblicher Personalüberhang entsteht.

- Untermuster 3c: Den Betrieben werden Sparmaßnahmen auferlegt, z. B.: da mir auf Grund der bei meiner Behörde durchzuführenden Sparmaßnahmen im Personalbereich gegenwärtig und auf absehbare Zeit keine freien Stellen zur Verfügung stehen.

- Untermuster 3d: Der Staat initiiert Einsparungen im Personalbereich, z. B.: dass, durch die vom Deutschen Bundestag beschlossenen Haushaltseinsparungen dem [...] im Personalbereich Einsparungen auferlegt sind, die die Berücksichtigung externer Bewerber zum jetzigen Zeitpunkt grundsätzlich ausschließen;

4. Muster 4: Es gibt zu viele/sehr viele Bewerber, z. B.: Bitte haben Sie Verständnis, dass wir wegen der großen Zahl an Einsendungen diesen Weg der Absage wählen müssen; eine Anzahl von Bewerbern impliziert zwangsläufig auch eine Vielzahl von Absagen; Gern hätte ich Ihnen eine noch detailliertere Rückmeldung zu Ihren 
Unterlagen gegeben, um den negativen Beigeschmack einer Absage etwas zu mildern. Ich bitte um Ihr Verständnis, dass mir dies bei der Vielzahl an Bewerbern nicht möglich war.

5. Muster 5: Es gibt Kandidaten, die den Anforderungen des Betriebs besser entsprechen, z. B.: weil es Mitarbeiter gibt, die unseres Erachtens besser mit dem von uns beschriebenen Anforderungsprofil harmonieren; bei mehreren gleich qualifizierten Bewerbern sind schon kleine Details ausschlaggebend; aufgrund der angespannten Konkurrenzsituation; Nach Prüfung der eingegangenen Bewerbungen müssen wir Ihnen heute leider mitteilen, dass Sie aufgrund stellenspezifischer Kriterien nicht in den engsten Kreis der Bewerber einbezogen werden konnten; Ihre Bewerbung kam leider nicht in die engere Auswahl, da andere Kandidatenprofile den speziellen Anforderungen der zur Diskussion stehenden Position näher kamen.

- Untermuster 5a: Der Bewerber ist zu alt, z. B.: Wie Sie aus dem aktuellen Merkblatt ersehen, ist das Höchstalter für Bewerber/innen um [...] bei [...] auf 30 Jahre festgelegt worden. Da Sie diese Altersgrenze überschritten haben, können wir Ihre Bewerbung leider nicht in das nächste Auswahlverfahren einbeziehen;

6. Muster 6: Die Stelle wurde an einen anderen Bewerber vergeben, z. B.: Leider müssen wir Ihnen mitteilen, dass wir die Stelle zwischenzeitlich an einen anderen Bewerber vergeben haben.

7. Muster 7: Im Moment stellen wir nicht ein, aber es ist möglich, dass wir uns bei Ihnen in Zukunft melden ${ }^{14}$, z. B.: Ich komme gerne auf Sie zurück; Ihr Bewerbungsschreiben lege ich gern auf Wiedervorlage, um zu gegebener Zeit darauf zurückgreifen zu können; Ihre Einwilligung vorausgesetzt, werden wir Ihre Bewerbung gerne in unserer Boxengasse parken. Bei einer freien Startposition werden wir uns mit Ihnen in Verbindung setzen; Sobald wir eine neue Kollegin suchen, werden wir uns bei Ihnen melden, versprochen!; Wir haben Ihr Profil elektronisch gespeichert.

8. Muster 8: Im Moment können wir nicht entscheiden, wir erstellen eine Bewerberliste, z. B.: Sie kommen auf unsere Bewerberliste;

9. Muster 9: Der Bewerbungsprozess ist noch nicht beendet ${ }^{15}$, z. B.: Die Bearbeitung/ die Auswertung der eingegangenen Bewerbungsunterlagen wird voraussichtlich einige Zeit in Anspruch nehmen; Das Auswahlverfahren wird einige Wochen in Anspruch nehmen und muss in unserem Institut mehrere Gremien durchlaufen;

- Untermuster 9a: Die Bearbeitung der Unterlagen dauert lange, z. B.: Die Vielzahl der eingehenden Bewerbungsunterlagen wird einige Zeit in Anspruch nehmen; Die Bearbeitung/die Auswertung der eingegangenen Bewerbungsunterlagen wird voraussichtlich einige Zeit in Anspruch nehmen; Das Auswahlverfahren wird einige Wochen in Anspruch nehmen und muss in unserem Institut mehrere Gremien durchlaufen;

\footnotetext{
${ }^{14}$ Dies v. a. in den Eisschreiben.

${ }^{15}$ Dies v. a. in den Aperitif-Briefen.
} 
- Untermuster 9b: Das Auswahlverfahren hat noch nicht angefangen, z. B.: Mit dem Auswahlverfahren kann jedoch erst begonnen werden, wenn die erforderlichen haushaltstechnischen Grundlagen geschaffen worden sind;

- Untermuster 9c: Die Unterlagen werden/wurden weitergeleitet, z. B.: Ich habe Ihre Bewerbungsunterlagen zuständigkeitshalber an [...] weitergeleitet.

\section{Schlussfolgerungen}

Die angeführten Begründungen können grob in vier Typen unterteilt werden. Einerseits liegen die Gründe für die Absage auf der Seite der jeweiligen von dem Bewerber angeschriebenen Firma, wozu sich auch die Absageschreiber bekennen. In solchen Begründungen werden jedoch objektive Tatsachen bestätigt, dass z. B. keine Einstellungen vorgenommen werden. Dabei wird sehr stark auf das Image der Firma geachtet.

Die zweite Gruppe bilden Begründungen der Absagen, die im Bereich der Wirtschaft zu suchen sind. Dabei wird die Schuld dem Staat und der Situation auf dem Arbeitsmarkt zugeschoben. Es wird jedoch nur Bezug auf objektive Tatsachen - Truismen - genommen, über die man in der Tagespresse lesen oder im Radio/Fernsehen hören kann. Da sie aber oft wiederholt und immer als Argumente angeführt werden, glaubt man, dass solche Truismen nolens volens zur Wahrheit werden. Es lässt sich aber bemerken, dass mit den Begründungen solcher Art auch eher spärlich umgegangen wird.

In der dritten Gruppe finden sich Begründungen, die im Zusammenhang mit dem Bewerber stehen. Es handelt sich dabei um dessen mangelnde Qualifikationen oder nicht entsprechende Qualifikationen. Zu einem Argument wird sogar die Feststellung, dass es sehr viele Bewerber gibt/gab. Als rechtswidrig soll die Begründung betrachtet werden, in der mit dem nicht entsprechenden Alter des Bewerbers argumentiert wird, denn ein Absageschreiben darf vor allem keinen Hinweis auf eines der acht in § 1 AGG genannten Kriterien (Rasse, ethnische Herkunft, Geschlecht, Religion oder Weltanschauung, Behinderung, Alter oder sexuelle Identität) enthalten ${ }^{16}$.

Beim Formulieren der Begründungen werden folgende Mittel gebraucht: Konstruktionen mit kausalen Präpositionen, z. B. aufgrund der Sparpolitik des Berliner Senats, wegen des bestehenden Einstellungsstopps; Nebensätze mit kausalen Subjunktoren, z. B. [...] da wir keine Online-Redaktion in Berlin haben. [...], [...] weil geeignete Arbeitsplätze nicht zur Verfügung stehen. [...]; Sätze mit dem Konjunktor denn, z. B. [...] denn bei uns - wie Sie sicher in den Medien verfolgt haben - herrscht ein weiterer Einstellungsstopp [...]; Deklarativsätze, z. B. [...] Wir haben uns für einige Kandidatinnen und Kandidaten entschieden, deren Profil noch genauer den Anforderungen der ausgeschriebenen Position entspricht [...].

${ }^{16} \mathrm{Vgl} . \mathrm{http}: / /$ www.azubee.de/fileadmin/user_upload/.pdf/azubee_allgemein/Bewerbern_diskriminierungsfrei_absagen.pdf [Zugriff: 23.07.2013]. 
Die anhand der Absageschreiben ermittelten Argumentationsmuster können als „kommunikative Muster“ (Günthner \& Knoblauch, 1994, S. 703), die typisch für diese Textsorte sind, betrachtet werden. Die im strukturellen Teil der Begründung in den Absageschreiben auftretenden Strukturen können daher als Realisierungen solcher kommunikativen Muster betrachtet werden, und demgemäß „Begründungsmuster“ genannt werden (vgl. Bubenhofer \& Scheurer, 2014, S. 251). Aufgrund ihrer hohen Frequenz in der behandelten Textsorte sind sie formelhaften Charakters, da die Notwendigkeit, auf eine Bewerbung eine Antwort zu geben, in jeder Firma zu den wiederkehrenden Handlungen des Geschäftsalltags gehört, die bewältigt werden müssen. Aus diesem Grunde ,verfestigt sich jede Handlung, die man häufig wiederholt, zu einem Modell, welches unter Einsparung von Kraft reproduziert werden kann und dabei vom Handelnden als Modell aufgefasst wird. Habitualisierung in diesem Sinne bedeutet, dass die betreffende Handlung auch in Zukunft ebenso und mit eben der Einsparung von Kraft ausgeführt werden kann“ (Berger \& Luckmann, 1980, S. 56, Hervorhebung - J.S.).

Das bestätigt auch Tappe (2002, S. 130), indem sie anführt: „Formelhafte Strukturen bilden ein Repertoire, auf das der Sprachteilhaber bei der Bewältigung kommunikativer Aufgaben sowohl im Produktions- als auch im Rezeptionsprozess zurückgreifen kann. Dies gilt auf allen Textebenen“".

Im vorliegenden Falle werden diese Bedürfnisse nach allen Regeln der Kunst und unter Berücksichtigung der Faktoren, die auch vom Gesetz her geregelt sind ${ }^{17}$, befriedigt. Aus dem Grunde greifen Absageschreiber sehr oft zu fertigen Vorlagen, mit denen sie diese jedoch unangenehme Aufgabe schnell, problemlos und routinemäßig erledigen können, zumal diese Begründungen aufgrund ihrer Vorkommenshäufigkeit in gewisser Hinsicht als ,erprobt“ und „bewährt“ aufgefasst werden können.

Die hohe Frequenz der Begründungen in den Absageschreiben kann in gewisser Hinsicht verwundern, zumal man unter vielen Ratschlägen zum Verfassen von Absageschreiben auch Warnungen davor findet, in Absagebriefen Begründungen anzuführen. Im Gegensatz dazu stehen im Duden (2005b, S. 129) Ratschläge, wo man folgende Empfehlungen finden kann: „Machen Sie sich die Mühe, eine Absage zu schreiben, die dem Bewerber einen Grund für die Absage nennt!“, denn - wie Straub (2014, S. 30) anführt - „Bewerber erwarten nachvollziehbare Gründe in Absageschreiben. Für Unternehmen ist das nicht einfach einzulösen. Doch wenn es ein Vorstellungsgespräch oder formale Gründe für die Absage gab, sollte das in das Absageschreiben eingebaut werden“".

\section{Literatur}

Berger, P.L. \& Luckmann, Th. (1980). Die gesellschaftliche Konstruktion der Wirklichkeit. Frankfurt am Main: Fischer Taschenbuch Verlag.

Brinker, K. (2010). Linguistische Textanalyse. Eine Einführung in Grundbegriffe und Methoden. 7. überarbeitete und erweiterte Auflage. Berlin: Erich Schmidt.

\footnotetext{
${ }^{17}$ Vgl. Allgemeines Gleichbehandlungsgesetz.
} 
Bubenhofer, N. \& Scheurer, P. (2014). Warum man in die Berge geht. Das kommunikative Muster „Begründen" in alpinistischen Texten. In U. Kleinberger \& K.S. Roth \& S. Hauser (Hrsg.), Textsorten und Textmuster im Wandel. Aktuelle Tendenzen der diachronen Text(sorten)linguistik (S. 245-273). Bern: Peter Lang.

Czachur, W. (2007). Textmuster im Wandel. Ein Beitrag zur textlinguistischen Erforschung der Vereinssatzungen im 19. Jahrhundert. Wrocław-Dresden: Atut \& Neisse Verlag.

Dąbrowska-Burkhardt, J. (2012). Argumentationsmuster des transnationalen EU-Reformvertragsdiskurses und ihre Wechselwirkung in der deutschen und polnischen Berichterstattung. In F. Grucza et al. (Hrsg.), Akten des XII. Internationalen Germanistenkongresses Warschau 2010 Vielheit und Einheit der Germanistik weltweit; Band 16. Warschau, Polska, 2010 (S. 33-38). Frankfurt am Main: Peter Lang.

Drescher, M. (1994). Für zukünftige Bewerbungen wünschen wir Ihnen mehr Erfolg. Zur Formelhaftigkeit von Absagebriefen. Deutsche Sprache, 22 (2), 117-137.

DUDEN. (1997). Briefe gut und richtig schreiben. Ratgeber für richtiges und modernes Schreiben. Mannheim-Leipzig-Wien-Zürich: Dudenverlag.

DUDEN. (2005a). Wzory listów niemieckich. Warszawa: Dudenverlag.

DUDEN. (2005b). Moderne Geschäftsbriefe leicht gemacht. Mannheim-Leipzig-Wien: Dudenverlag.

Ehlich, K. \& Rehbein, J. (1986). Muster und Institution. Tübingen: Gunter Narr Verlag.

Gałczyńska, A. (2003). Akty odmowy we wspótczesnym języku polskim. Kielce: Wydawnictwo Akademii Świętokrzyskiej.

Gohl, Ch. (2006). Begründen im Gespräch. Tübingen: Niemeyer.

Günthner, S. \& Knoblauch, H. (1994). „Forms are the food of faith“: Gattungen als Muster kommunikativen Handelns. Kölner Zeitschrift für Soziologie und Sozialpsychologie, 46 (4), 693-723.

Hayashi, A. (1997). BITTEN und ABSAGEN im Gespräch - Reaktion der Japaner auf die deutschen Strategien. Bulletin of Tokyo Gakugei University, Sekt. II Humanities, 48, 109-121.

Herbig, A.F. (1992). ,, Sie argumentieren doch scheinheilig! “. Sprach- und sprechwissenschaftliche Aspekte einer Stilistik des Argumentierens. Frankfurt am Main: Peter Lang.

Hovermann, C. (2009). Stilvolle Absagen im Geschäftsalltag: Wie Sie negative Informationen freundlicher und konstruktiv vermitteln. Bonn: Verlag Dt. Wirtschaft.

Itakura, U. (2001). Empfehlen und Absagen. Verkaufsgespräche von Deutschen und Japanern im Vergleich. In J. Fürnkäs et al. (Hrsg.), Zwischenzeiten - Zwischenwelten. Festschrift für Kozo Hirao (S. 375-385). Frankfurt am Main: Peter Lang.

Itakura, U. (2004). Absagen bei der Einkaufs-/Verkaufshandlung im Deutschen und Japanischen. Versuch einer pragmatischen Interpretation. Deutsch als Fremdsprache, 4, 220-226.

Lüger, H.-H. (1992). Sprachliche Routinen und Rituale. Frankfurt am Main: Peter Lang.

Müller, E. \& Moser, K. (2006). Reaktionen auf Ablehnungsschreiben an Bewerber. Das Beispiel „Eisschreiben“. Zeitschrift für Personalforschung, 20 (4), 343-355.

Nęcki, Z. (1996). Komunikacja międzyludzka. Kraków: Antykwa.

Perelman, Ch. (1979). Logik und Argumentation. Königstein im Taunus: Athenäum.

Sáfár, É. (1999). Sprachliche Argumentationsstrategien deutscher und ungarischer Presseorgane im Wahljahr 1994. Ein Modell zur Analyse persuasiver Texte. In M. Hoffmann \& Ch. Kessler (Hrsg.), Beiträge zur Persuasionsforschung unter besonderer Berücksichtigung textlinguistischer und stilistischer Aspekte (S. 101-119). Berlin: Peter Lang.

Schröder, Th. (2003). Die Handlungsstruktur von Texten. Ein integrativer Beitrag zur Texttheorie. Tübingen: Narr Francke Attempto.

Straub, R. (2014). Absagen mit Stil und Respekt. Personalmagazin, 11, 30.

Szczęk, J. (2015). Absageschreiben auf Bewerbungen. Eine pragmalinguistische Studie. Berlin: Frank \& Timme. 
Tappe, S. (2002). Formelhaftigkeit und Argumentation. In S. Bastian \& F. Hammer (Hrsg.), Aber, wie sagt man doch so schön... Beiträge zu Metakommunikation und Reformulierung in argumentativen Texten (S. 107-143). Frankfurt am Main: Peter Lang.

Unwerth, H.-J. von \& Buschmann, U. (1981). Konfliktive Sprechakte und Lehrwerktext. In B.-D. Müller \& R. Paris (Hrsg.), Konfrontative Semantik (S. 60-71). Tübingen: Lexika Verlag.

Wengeler, M. (2003). Topos und Diskurs. Begründung einer argumentationsanalytischen Methode und ihre Anwendung auf den Migrationsdiskurs (1960-1985). Tübingen: de Gruyter.

Wierzbicka, A. (1987). English Speech Act Verbs. A Semantic Dictionary. Sydney: Emerald Group Pub.

\section{Internetquellen}

http://www.azubee.de/fileadmin/user_upload/.pdf/azubee_allgemein/Bewerbern_diskriminierungsfrei_absagen.pdf [Zugriff: 23.07.2013]

http://www.duden.de/rechtschreibung/Aperitif [Zugriff: 14.11.2015]

http://www.duden.de/rechtschreibung/Eis_Wasser_Speise [Zugriff: 14.11.2015]

http://www.manager-magazin.de/koepfe/karriere/0,2828,321132,00.html [Zugriff:4.11.2007] 